\title{
Can Therapeutic Ultrasound Accurately Detect Bone Stress Injuries in Athletes
}

Papalada A, Malliaropoulos N, Tsitas K, et al. Ultrasound as a primary evaluation tool of bone stress injuries in elite track and field athletes. Am J Sports Med. 2012;40:915-919.

\section{Objective}

To evaluate therapeutic ultrasound (TUS) for the primary detection of bone stress injuries in symptomatic elite track and field athletes.

\section{Design}

Comparison of TUS with magnetic resonance imaging (MRI) as the diagnostic standard. Both the symptomatic and the nonsymptomatic lower extremity were examined.

\section{Setting}

From 2000 to 2010, 113 elite track and field athletes were assessed at a sport medicine center in Greece.

\section{Patients}

Athletes with a clinically suspected bone stress injury, ie, unilateral exercise-induced lower extremity pain of $<1$ month's duration that improved with rest, with tenderness limited to a focal area, and with no history of trauma, who were diagnosed by a single sport medicine physician, were eligible. Exclusion criteria were suspected muscle strain, tendinopathy, compartment syndrome, or low back pain. The mean age of the 113 patients was 20 years and $53 \%$ were women.

\section{Description of test and diagnostic standard}

One physical therapist used a high resolution Sonopuls 434 (Enraf-Nonius, Rotterdam, the Netherlands) device to perform TUS (the test) on the symptomatic area of the lower extremity, at an intensity of $2.00 \mathrm{~W} / \mathrm{cm}^{2}$ and a frequency of $1 \mathrm{MHz}$, with the most symptomatic point spotted for 30 seconds, at reduced intensity if there was pain. The results were classified as no pain negative or pain - positive. A Tesla system with a 1.5-T scanner was used to perform MRI (the 
diagnostic standard). Axial and coronal images were obtained by a protocol that facilitated the assessment of periosteal and bone marrow edema. Bone stress was graded 0 (normal appearance), 1-3 (increasing severity of edema), or 4 (visible fracture line on MRI). The images were read in random order by 1 musculoskeletal radiologist blinded to clinical and TUS findings. Grades 1-4 constituted a bone stress injury. The TUS and MRI examinations were performed <1 month after the onset of the patients' symptoms, with the time interval between TUS and MRI, 04 days.

\section{Main outcome measure}

The primary outcome measures were sensitivity, specificity, and positive and negative predictive values for TUS in comparison with MRI results.

\section{Main results}

Grades 0 to 4 bone stress injuries were identified by MRI in 3,12, 15, 77, and 6 patients, respectively, with multiple injuries in 5 of the 113 athletes. At TUS no injury was found in 22 of 113 patients; 2 with grade 0 injuries, 8 with grade 1,8 with grade 2 , and 4 with grade 3 injuries on MRI. Sensitivity of TUS for the presence of a bone stress injury was $82 \%$ overall, but $44 \%$ for grades 1 and 2 injuries. Specificity was 67\%, positive predictive value was 99\%, negative predictive value was $13 \%$, and overall accuracy was $81 \%$. In contralateral testing of the uninjured leg, MRI identified 1 grade 1 injury, and TUS found 3 positive results.

\section{Conclusions}

The authors reported that therapeutic ultrasound, tested against MRI findings, had high accuracy in identifying grade 3 and 4 bone stress injuries of the lower extremity, but its sensitivity was poor for less severe injuries. There were too few patients without stress injuries to validly test the specificity of TUS.

Source of funding for the original study: The authors reported no conflicts of interest.

Correspondence about the original article: Nicola Maffulli, MD, MS, PhD, FRCS(Orth), Centre for Sports and Exercise Medicine, Barts and The London School of Medicine and 
Dentistry, Mile End Hospital, 275 Bancroft Road, London E1 4DG, England (n.maffulli@qmul.ac.uk).

\section{Commentary}

Bone stress injuries are problematic from two perspectives. First, they are often painful enough to limit or halt athletic performance and require a period of rest to resolve; and second, the amount of rest required cannot be predicted because injury severity cannot be reliably determined from existing systems of clinical or radiological grading, ${ }^{1}$ The attempt of Papalada et al to identify a reliable diagnostic tool for bone stress injuries is clinically important because both diagnostic false negatives and false positives can be disastrous for elite performance, by either placing athletes at risk of increasing the severity of injury or needlessly disrupting training at critical times.

Diagnostic cohort studies of bone stress injuries are inherently challenging because of a relatively low incidence of injury. The authors approached the problem by recruiting participants with a heterogeneous sample of bone stress injuries. However, this approach does not account for the likelihood that some anatomical locations (navicular) will be more sensitive to TUS than others (femur). Previous reports suggest that when single location bone stress injuries are examined, ultrasound can have very low diagnostic sensitivity and specificity. ${ }^{2,3}$ It may be that grouping different injury sites for analysis may actually obscure individually favorable applications.

The authors adopted MRI as the gold standard for comparison with TUS, but they interpreted the MRI grading system that they used relatively loosely. ${ }^{4}$ The inclusion of bone marrow edema as a characteristic of grade 1 bone stress injury differs from Fredericson's original system (Fredericson's definition is "Mild-to moderate periosteal edema on T2-weighted images only, with no focal bone marrow abnormality" ${ }^{4}$ versus Papalada's "periosteal and bone marrow edema.") The authors also fail to note that Fredericson's system was developed specifically for tibial stress injuries. For these reasons, their analyses of TUS outcomes in relation to MRI severity grades are difficult to interpret.

The conclusion of Papalada et al that "...TUS is a reliable, reproducible procedure, easy to perform in an office setup or at the pitch side" is not entirely representative of the nearly $20 \%$ 
of false negatives reported, nor the particularly poor sensitivity of TUS for low grade injuries. Although the data, as collected, are insufficient to support the authors' conclusions, the appealing cost and utility of TUS justifies further investigation. Of particular clinical significance however, is the elephant in the room - the fact that the TUS procedure is based on producing pain at the site of injury while other diagnostic imaging modalities are essentially painless. Patient feedback with respect to test acceptability will be an important outcome measure in future work.

Belinda Beck, PhD, MS, BHMS(Ed)

School of Rehabilitation Sciences

Griffith University

Gold Coast, Queensland, Australia

\section{References}

1. Beck BR, Bergman AG, Miner M, et al. Tibial stress injury: Relationship of radiographic, nuclear medicine bone scanning, MR imaging, and CT severity grades to clinical severity and time to healing. Radiology. 2012;263:811-818.

2. Boam WD, Miser WF, Yuill SC, et al. Comparison of ultrasound examination with bone scintiscan in the diagnosis of stress fractures. J Am Board Fam Pract. 1996;9:414-417.

3. Romani WA, Perrin DH, Dussault RG, et al. Identification of tibial stress fractures using therapeutic continuous ultrasound. J Orthop Sports Phys Ther. 2000;30:444-452.

4. Fredericson M, Bergman G, Hoffman KL, et al. Tibial stress reaction in runners. Correlation of clinical symptoms and scintigraphy with a new magnetic resonance imaging grading system. Am J Sports Med. 1995;23:472-481. 